\title{
Single-Pulse Harmonic Modulation for Short Range Biomedical Inductive Data Transfer
}

\author{
Matthew Schormans, Virgilio Valente, and Andreas Demosthenous \\ Department of Electronic and Electrical Engineering, University College London, London WC1E 7JE, United Kingdom. \\ Email: \{matthew.schormans.10; v.valente; a.demosthenous\}@ucl.ac.uk
}

\begin{abstract}
Short-range, low-power, high data-rate telemetry is an increasingly desirable feature for implantable medical devices (IMDs), and is commonly implemented using an inductive link. Pulse Harmonic Modulation (PHM) provides the desired high data rates and low power consumption, but requires precise pulse timing. This paper presents a modification of PHM, SinglePulse Harmonic Modulation (SPHM), which offers reduced power consumption and lower implementation complexity. In order to test the SPHM concept, transmitter and receiver circuits were designed in $0.35 \mu \mathrm{m}$ CMOS and simulated. The simulated results suggest that the circuits can transceive data at $50 \mathrm{Mb} / \mathrm{s}$, consuming $1.49 \mathrm{pJ} / \mathrm{b}$ and $2.59 \mathrm{pJ} / \mathrm{b}$ at the transmitter and receiver respectively, from a $1.2 \mathrm{~V}$ supply.
\end{abstract}

\section{INTRODUCTION}

Short range inductive data telemetry is a key feature of modern implantable medical devices (IMDs). The data rate required for a given IMD however will depend on application. For instance, an implanted stimulator may only require a few $\mathrm{kb} / \mathrm{s}$ of data rate, such that it can be programmed, whereas a multi-channel neural recording device may require many $\mathrm{Mb} / \mathrm{s}$ to transmit the neural data. This is particularly relevant for emerging neural recording and ECoG systems with high channel count [1], [2]; as the number of channels increase, the data rate must also increase [3]. Other IMDs requiring high data rates include cochlear implants and visual prostheses.

Despite this growing requirement for robust data telemetry, the power consumption of any data telemetry scheme must also be tightly controlled, since IMDs generally have only small power budgets available. Additionally IMDs are generally small in size, limiting implementation size/complexity. As a result, this compromise drives the design process of most data telemetry schemes used in IMDs.

An attractive feature of inductive data telemetry is the ability to superimpose data telemetry onto a power link, using techniques like passive phase shift keying (PPSK) [4]. While a combined power/data link is attractive for a compact system, the data rate is usually limited by the power carrier frequency. Such systems therefore rarely exceed data rates of a few $\mathrm{Mb} / \mathrm{s}$. Such low data rates are unsuitable for transmitting high volumes of recorded data.

To achieve higher data rates it is necessary to employ a dedicated data link, which can safely use a higher carrier frequency, since power transfer efficiency is no longer an issue. Such systems can then employ conventional data modulation schemes such as FSK or QPSK [5]. While providing an improved data rate, these systems still require a carrier to be generated, which can be a concern when trying to conserve power.
Pulse Harmonic Modulation (PHM) is an example of a carrier-less data telemetry scheme that takes advantage of the resonant nature of an inductive link to achieve higher data rates and lower power consumption than other inductive methods [6], [7], [8]. However, implementing PHM requires a pulse-pattern generator that must create a pair of pulses with a precise delay (sub-ns precision) and precise amplitude. Deviation from the optimal delay and amplitude values can result in inter-symbol interference and have a negative impact on performance.

This paper presents a single-pulse PHM implementation (SPHM) that retains the benefits of PHM (low power consumption, high data rate, no carrier required), while reducing the required circuit implementation complexity, reducing the sensitivity to process, voltage, and temperature variations (PVT), and further reducing the power consumption. A transmitter and receiver circuit have been designed and simulated in $0.35 \mu \mathrm{m}$ CMOS to verify the concept.

The paper is structured as follows: Section II briefly reviews the principle of PHM and presents the SPHM approach, Section III covers the design of the transmitter and receiver circuits, Section IV presents the simulated circuit behaviour, and Section $\mathrm{V}$ concludes the paper.

\section{THEORY}

\section{A. Pulse Harmonic Modulation}

The theory of operation of PHM is first described by Inanlou et. al. [6], and is briefly summarised here. The key behind PHM involves taking advantage of the resonant impulse response provided by relatively high-Q inductive links. Driving such a link with a sharp impulse will generate an oscillation with a rapid rise and slow decay. By carefully generating an opposing 'suppression' impulse with the correct delay and amplitude, an opposing ringing response is generated, cancelling

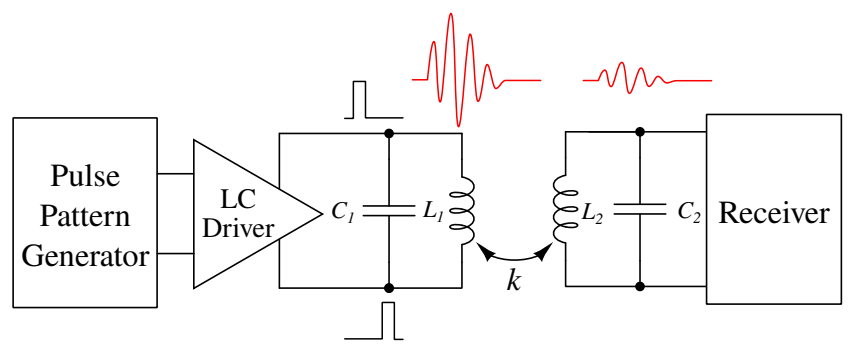

Fig. 1: Block diagram of a PHM data link, showing excitation and suppression pulses. 

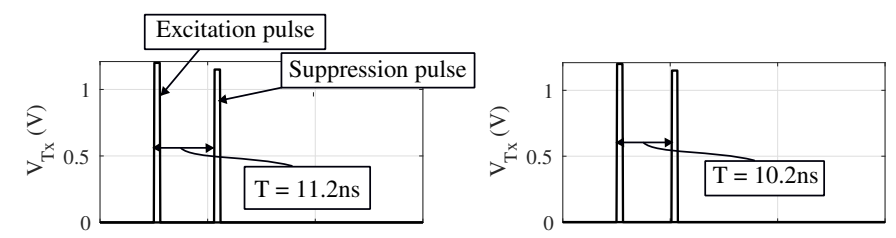

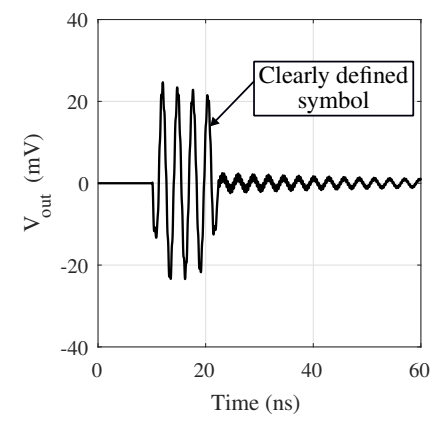

(a)

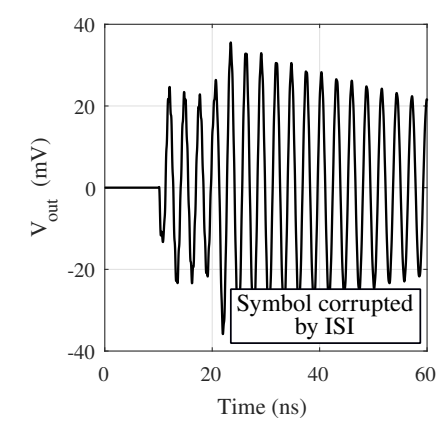

(b)
Fig. 2: Simulation of ISI in PHM for different interpulse delays, (a) $11.2 \mathrm{~ns}$, (b) $10.2 \mathrm{~ns}$. The link resonant frequency here is $\approx 350 \mathrm{MHz}$.

the slow decay and leaving a short burst of oscillation [8]. These short bursts can be used to form a bitstream with a much higher data rate than if the oscillations were left to decay naturally. Fig. 1 shows a block diagram of a typical PHM data link, consisting of a pulse pattern generator, two coupled coils, and an RF receiver. The parallel capacitances shown $\left(C_{1}, C_{2}\right)$, can be formed from system parasitics or by additional capacitors, depending on the receiver bandwidth. Another benefit of PHM is that the receiver requires no local oscillator or mixer to acquire the data, simplifying the circuit design and reducing power consumption.

The key shortfall of this scheme, however, is its sensitivity to the delay and amplitude of the excitation and suppression pulses. Should they vary from optimum values the data can suffer significant inter-symbol interference (ISI), leading to corruption of data bits. This is illustrated in Fig. 2; the correct pulse timing in this example is determined to be 11.2ns, Fig. 2 a shows a well defined symbol. However introducing a $1 \mathrm{~ns}$ error (Fig. 2b) renders the transmitted bit completely hidden by ISI.

This sensitivity to pulse timing must be taken into consideration when designing PHM circuits in CMOS. For instance, PVT variations could easily create wide variations in pulse timing unless techniques such as digital trimming and autocalibration are used, which add further complexity and cost.

\section{B. Single-Pulse Harmonic Modulation}

SPHM modifies PHM, by replacing the precisely timed suppression pulse with a long pulse applied to a 'damping' switch connected across the coils of the link. In this way the symbol is shortened without the need for the interpulse delay to be precise. Fig. 3 shows a basic block diagram of an SPHM system.

The theory of operation of SPHM is described below, and is illustrated in Fig. 4. The incoming data is assumed to be

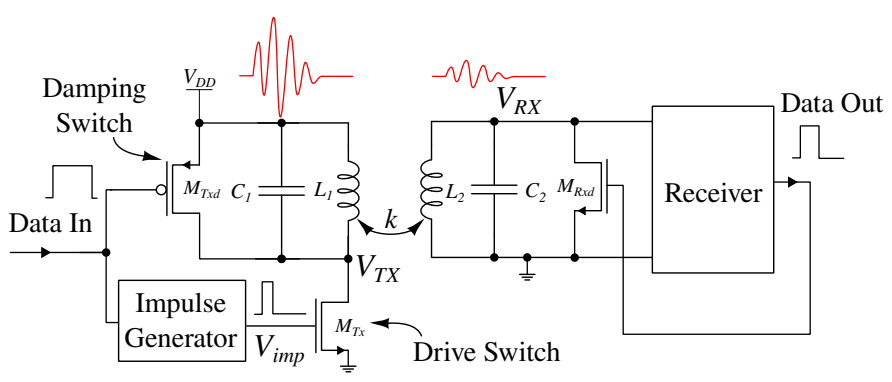

Fig. 3: Block diagram of the SPHM scheme.

between $20 \%$ and $50 \%$ high to encode a ' 1 ', and $100 \%$ low to encode a ' 0 '. This restriction is necessary as the data stream is in direct control of the transmit damping switch $M_{T x d}$. The incoming data also directly drives an impulse generator, which should generate sharp impulses on the rising data edge to drive the transmitter switch $M_{T x}$. This impulse must be short in comparison to the natural frequency of the resonant circuit formed by $L_{1}$ and $C_{1}$. The maximum impulse width, $t_{p w}$, is defined as [6]:

$$
t_{p w(\max )}=2 \sqrt{2} \sqrt{L_{1} C_{1}}
$$

Defining the impulse width to be sufficiently short (i.e. $t_{p w} \leq t_{p w(\max )}$ according to (1)) allows the short pulse to be approximated as an ideal impulse. These impulses trigger the switch $M_{T x}$, drawing a burst of current through the tank formed by $L_{1}$ and $C_{1}$. This will create a sharply rising ringing response, which is detected in the receiver side by $L_{2}$ and $C_{2}$. The switch $M_{T x d}$ will turn on once the data input returns low. This has the effect of reducing the Q-factor of $L_{1}$ and $C_{1}$ greatly (the $r_{\mathrm{on}}$ of $M_{T x d} \ll R_{\mathrm{P} 1}$ where $R_{\mathrm{P} 1}$ is the parallel loss resistance of $L_{1} \| C_{1}$ ). This reduction in $\mathrm{Q}$ causes the oscillations to rapidly be damped out at the primary side after

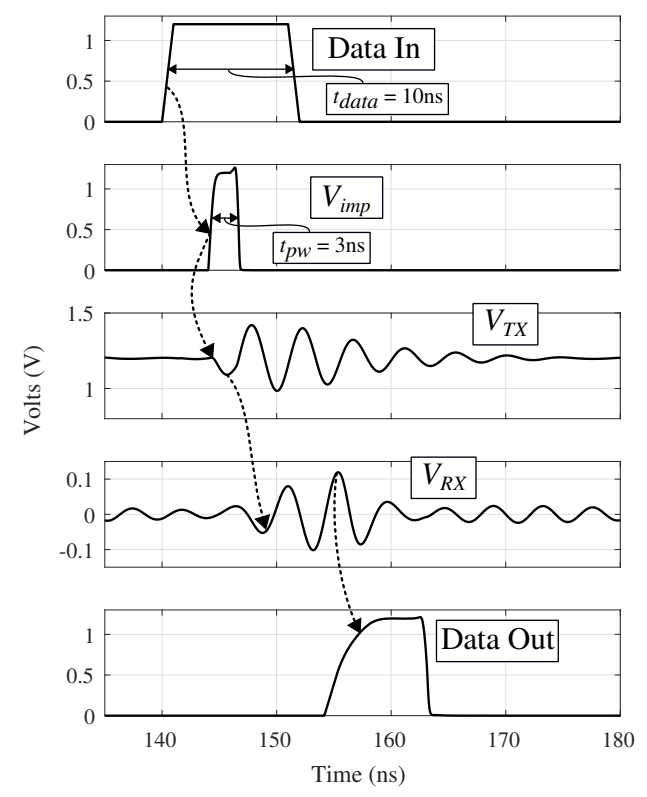

Fig. 4: Example timing diagram for SPHM. 


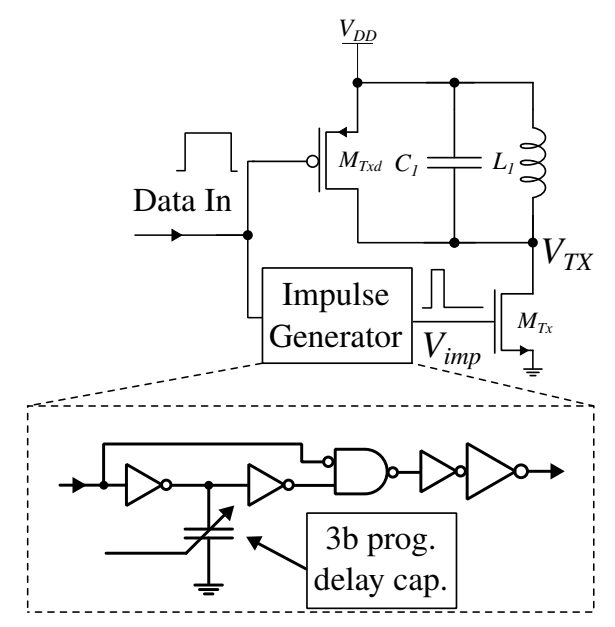

Fig. 5: Block diagram of the transmitter circuit.

a bit has been transmitted. The same principle is employed at the receiver. When no bit has yet been detected, the receiver damping switch $M_{R x d}$ is open, so the high $\mathrm{Q}$ of $L_{2} \| C_{2}$ allows the ringing signal to build up. Once the bit has been successfully detected, the receiver drives the gate of $M_{R x d}$ to short out the receiver input; damping the signal in the same way as $M_{T x d}$ at the transmitter.

Fig. 4 shows that the SPHM scheme exhibits the same short oscillatory bursts that characterise PHM, allowing for carrierless, relatively high rate data transmission. The key benefits of SPHM are as follows: since only one high speed impulse needs to be generated instead of two, the power consumption of the transmitter is reduced. Secondly, there is no strict requirement for precise pulse timing, other than the restriction that the impulse width $t_{p w}$ must be sufficiently short. The details regarding power consumption and resilience to timing variations are discussed in more detail in Section IV.

\section{Circuit Design}

To test the SPHM concept, transmitter and receiver circuits were designed in $0.35 \mu \mathrm{m} \mathrm{CMOS}$. These circuits are currently under fabrication, and their designs are discussed below.

\section{A. Transmitter}

The transmitter is a simple circuit, formed of the transistors $M_{T x}, M_{T x d}$, and an impulse generator. Fig. 5 shows the transmitter circuit in more detail.

The impulse generator shown in Fig. 5 functions by combining a programmable delay cell with a simple logic block. The data input is compared with a delayed version of that data; the output is only high when the input data is high and the delayed data is low. As such the pulse width $t_{p w}$ can be modified by altering the capacitance of the $3 \mathrm{~b}$ programmable capacitor (40fF, 80fF, 160fF). The output inverter chain is tapered to strongly drive the gate of $M_{T x}$.

\section{B. Receiver}

A block diagram of the receiver circuit is shown in Fig. 6. The core of the receiver circuit is a comparator based on

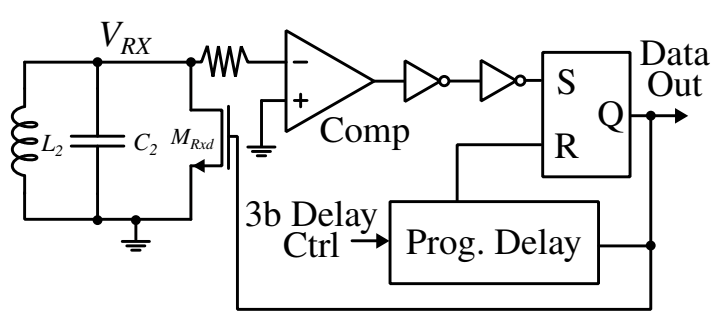

Fig. 6: Block diagram of the receiver circuit.

TABLE I: Link specifications for simulation in accordance with the link in Fig. 1. $R_{1}, R_{2}$ are the series losses for $L_{1}, L_{2}$

\begin{tabular}{|c|c|}
\hline$L_{1}, L_{2}$ & $200 \mathrm{nH}$ \\
\hline$C_{1}, C_{2}$ & $1 \mathrm{pF}$ \\
\hline$R_{1}, R_{2}$ & $5 \Omega$ \\
\hline$k$ & 0.1 \\
\hline
\end{tabular}

cross-coupled common-gate amplifiers [9], which are ideal for a ground referenced configuration. The operation of the circuit is such that the comparator output will remain low until a large enough deviation is present at $V_{R X}$, at which point the comparator output will trigger. This triggering sets the output latch, and activates the receiver damping switch $M_{R x d}$. The latch will reset itself after a delay defined by the programmable delay block. The programmable delay block uses a series of stacked delay cells as used in the transmitter in order to allow for longer length recovered bits. The series resistor at the comparator input is small $(500 \Omega)$ and serves two purposes. Firstly it provides some isolation between the reset switch and the comparator input, useful in this case as this type of comparator has a relatively low input impedance. Secondly it effectively provides a small offset at the input of the comparator, to reduce the chance of false triggering.

\section{Simulation Results}

The behaviour of the circuit was simulated to determine its data rate capabilities, limits of acceptable data pulse widths, and power consumption of the transmitter and receiver blocks. The results of these simulations are presented in this section.

The transmitter and receiver circuits were simulated on either side of a link with parameters as specified in Table I. These parameters result in a natural resonant frequency $f_{\text {res }} \approx$ $355.8 \mathrm{MHz}$. The simulations show a natural resonant frequency closer to $222 \mathrm{MHz}$, due to the additional capacitances seen from the transmitter and receiver interfaces. This frequency would likely be reduced further in the fabricated chip, which would be subject to further parasitic capacitances in the packaging and board. The precise natural frequency is not crucial however, as long as it is sufficiently higher than the data rate so that symbols are recognisable.

The results of a postlayout system-level simulation are shown in Fig. 7, where a pseudorandom sequence of data is transmitted and received, with a nominal data rate of 50Mbps.

The oscillatory bursts in $V_{T X}$ and $V_{R X}$ in Fig. 7 can be seen to have essentially no dead time between them in the case of two consecutive ' 1 's, indicating that $50 \mathrm{Mbps}$ is near 

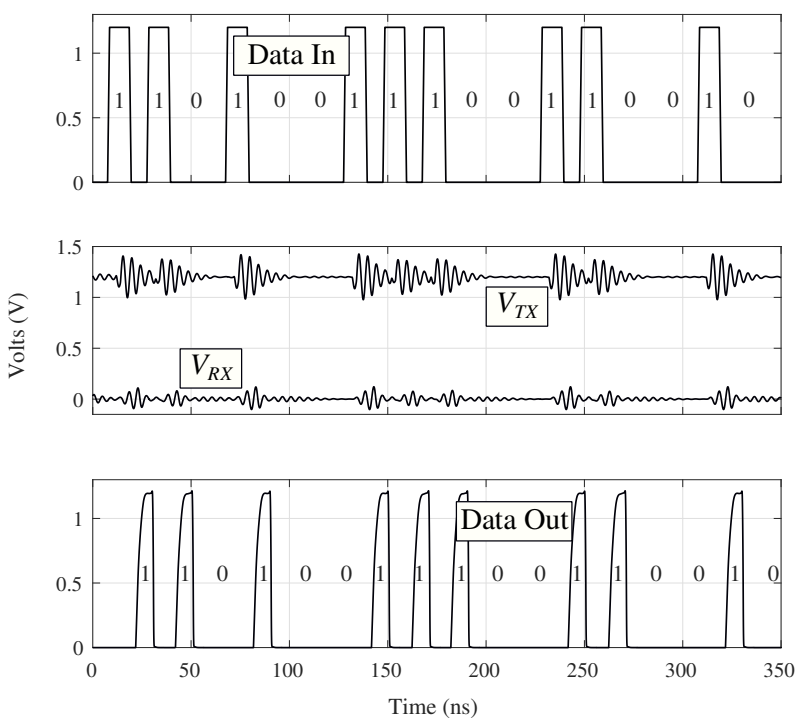

Fig. 7: Simulated timing data for transmission of a pseudorandom sequence at $50 \mathrm{Mbps}$.

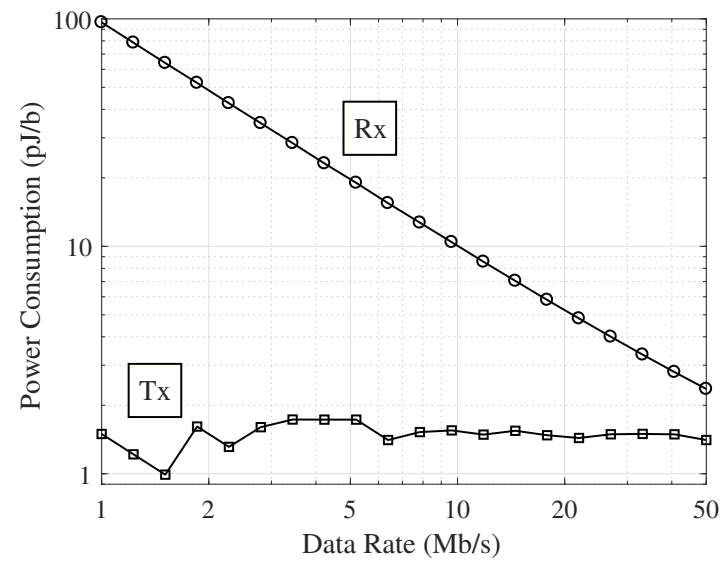

Fig. 8: Power consumption $(\mathrm{pJ} / \mathrm{b})$ vs increasing data rate.

the maximum data rate limit for this simulated system. Fig. 8 shows the power consumption in terms of energy per bit $(\mathrm{pJ} / \mathrm{b})$ against varying data rate. The data stream used was a stream of consecutive ' 1 's, as this should be the worst case for power consumption. Since the transmitter has very little quiescent power consumption, it maintains an almost constant value averaging at $\approx 1.49 \mathrm{pJ} / \mathrm{b}$. The receiver power consumption is dominated by quiescent consumption (mostly in the biasing for the comparator), and therefore presents a logarithmic rolloff as the data rate increases. The receiver therefore effectively works more efficiently at higher data rates, achieving $2.59 \mathrm{pJ} / \mathrm{b}$ at $50 \mathrm{Mb} / \mathrm{s}$, compared to $96.52 \mathrm{pJ} / \mathrm{b}$ at $1 \mathrm{Mb} / \mathrm{s}$. The power consumption figures here exclude the power drawn by pad buffers.

To determine the resilience of the system to variations in pulse width/timings, the system was tested with a sequence of consecutive ' 1 's at $50 \mathrm{Mb} / \mathrm{s}$, and the pulse width of the incoming data stream $\left(t_{\text {data }}\right)$ was varied from the nominal 10ns (50\% duty). Additionally Monte Carlo variations of the impulse width were simulated, to examine the effect of variations from the nominal pulse width $t_{p w}=3 \mathrm{~ns}$. The system was observed to successfully transceive in simulations as long as: $3 \mathrm{~ns} \leq t_{\text {data }} \leq 11 \mathrm{~ns}$ and $2 \mathrm{~ns} \leq t_{p w} \leq 3.8 \mathrm{~ns}$. This is in line with (1); $t_{\text {data }}$ only needs to be long enough to allow the link to start ringing, but if it is too long ISI will result. Similarly $t_{p w}$ must remain sufficiently short or the generated ringing will have a reduced amplitude.

\section{CONClusion ANd Future Work}

The SPHM method of data transmission through an inductive link has been presented, which improves upon traditional PHM. Transmitter and receiver circuits have been designed in $0.35 \mu \mathrm{m}$ CMOS to test the concept, which are currently under fabrication. Simulations of these circuits suggest the method is capable of $50 \mathrm{Mb} / \mathrm{s}$ data transmission, with a low power consumption.

These simulation results suggest that SPHM is well suited to tackle the increasing demand for higher data rates at lower power consumptions put forward by next-generation multichannel IMDs [3].

Future work will include comparison of chip measurements with simulation results, as well as development of a rigorous theoretical analysis to facilitate a quantitative optimization procedure.

\section{REFERENCES}

[1] S. A. Mirbozorgi, H. Bahrami, M. Sawan, L. A. Rusch, and B. Gosselin, "A Single-Chip Full-Duplex High Speed Transceiver for Multi-Site Stimulating and Recording Neural Implants," IEEE Transactions on Biomedical Circuits and Systems, vol. 10, no. 3, pp. 643-653, June 2016.

[2] R. Muller, H. P. Le, W. Li, P. Ledochowitsch, S. Gambini, T. Bjorninen, A. Koralek, J. M. Carmena, M. M. Maharbiz, E. Alon, and J. M. Rabaey, "A Minimally Invasive 64-Channel Wireless ECoG Implant," IEEE Journal of Solid-State Circuits, vol. 50, no. 1, pp. 344-359, Jan 2015.

[3] U. Bihr, T. Liu, M. Ortmanns, H. Xu, and J. Handwerker, "Telemetry for Implantable Medical Devices," IEEE Solid-State Circuits Magazine, pp. 47-51, 2014.

[4] D. Jiang, D. Cirmirakis, M. Schormans, T. Perkins, N. Donaldson, and A. Demosthenous, "An Integrated Passive Phase-Shift Keying Modulator for Biomedical Implants With Power Telemetry Over a Single Inductive Link," IEEE Transactions on Biomedical Circuits and Systems, vol. 11, no. 1, pp. 64-77, 2016.

[5] G. Simard, M. Sawan, and D. Massicotte, "High-speed OQPSK and efficient power transfer through inductive link for biomedical implants," IEEE Transactions on Biomedical Circuits and Systems, vol. 4, no. 3, pp. 192-200, 2010.

[6] F. Inanlou and M. Ghovanloo, "Wideband Near-Field Data Transmission using Pulse Harmonic Modulation," IEEE Transactions on Circuits and Systems I: Regular Papers, vol. 58, no. 1, pp. 186-195, 2011.

[7] F. Inanlou, M. Kiani, and M. Ghovanloo, "A 10.2 Mbps Pulse Harmonic Modulation Based Transceiver for Implantable Medical Devices," IEEE Journal of Solid-State Circuits, vol. 46, no. 6, pp. 1296-1306, 2011.

[8] M. Kiani and M. Ghovanloo, "A 20-Mb / s Pulse Harmonic Modulation Transceiver for Wideband Near-Field Data Transmission," IEEE Transactions on Circuits and Systems II: Express Briefs, vol. 60, no. 7, pp. 382-386, 2013.

[9] S. Guo and H. Lee, "An Efficiency-Enhanced CMOS Rectifier With Unbalanced-Biased Comparators for Transcutaneous-Powered HighCurrent Implants," IEEE Journal of Solid-State Circuits, vol. 44, no. 6, pp. 1796-1804, 2009. 